\title{
High mobility group box 1 in diabetic nephropathy (Review)
}

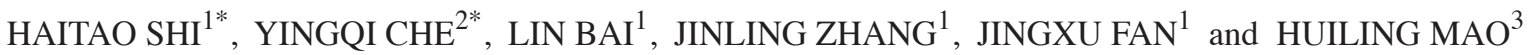 \\ Departments of ${ }^{1}$ Nephrology, ${ }^{2}$ Hematological Oncology and ${ }^{3}$ Science and Education, \\ Longnan Hospital, Daqing, Heilongjiang 163000, P.R. China
}

Received April 6, 2017; Accepted July 11, 2017

DOI: $10.3892 /$ etm.2017.4826

\begin{abstract}
Type 2 diabetes (T2D) is a complex disorder caused by the combined effects of genetic inheritance and environmental factors. The abnormal secretion of albumin via urine is the characteristic feature of a diabetic nephropathy (DN) patient. Moreover, the detection of this observable characteristic feature of $\mathrm{DN}$ is quite late. As a result the time, at which DN is observable, large extent of kidney damage has already occurred. Thus, this late observation significantly decreases the chances of efficient management of DN and associated outcomes. The current biomarker used to detect DN is microalbuminuria, the presence of albumin in the urine. However, the current biomarkers often lead to false negative results. The high mobility group box (HMGB)1 is an upcoming molecule being explored for its application in the management of DN. The present review enlightens the current status of HMGB1 in DN.
\end{abstract}

\section{Contents}

1. Introduction

2. Current views of DN

3. Serum creatinine levels during DN

4. HMGB1

5. HMGB1, DN and RAGE

6. HMGB1 signaling and DN

7. AGE and DN

8. Conclusions

\section{Introduction}

The clinical observance of significant loss in the glomerular filtration rate (GFR) is a direct indicator of diabetic

Correspondence to: Ms. Huiling Mao, Department of Science and Education, Longnan Hospital, 35 Aiguo Road, Ranghulu, Daqing, Heilongjiang 163000, P.R. China

E-mail: mao_hl1@163.com

${ }^{*}$ Contributed equally

Key words: diabetic nephropathy, high mobility group box 1, biomarkers nephropathy (DN) and is also associated with urine albumin excretion (1). Furthermore, DN is the most common causative agent for end-stage renal disease (ESRD). ESRD is also the result of type 2 diabetes (T2D) but is treatable. On the whole, in the United States, DN is the more prevailing cause of ESRD as compared to T2D.

DN is associated with high mortality as well as high morbidity rates during cardiovascular disease, long before ESRD (2). At present, many studies have explored DN extensively leading to information useable for the identification of DN at quite an early stage. However, the exact knowledge of pathophysiology of DN is still pending. Most of the confirmed factors identified for detection of DN include non-modifiable risk factors such as ethnicity, family history and elevated GFR early after diagnosis of T2D have been proven in many studies. Risk factors for developing DN that could be modified are glycemia, hypertension, smoking status, obesity and dyslipidemia. Research has shown that if the patients take good care of the modified risk factors such as glycemic controls, the progress of developing DN could be delayed.

\section{Current views of DN}

The state of untreated DN is the prime initiator of ESRD (3). The current biomarker used to detect DN is microalbuminuria, indicating extent of albumin secretion by the body via urination (4). The above technique involves recording of albumin-to-creatinine ration most probably in the first urine of the affected patient. The process of recording above ratio continues for $24 \mathrm{~h}$ at regular intervals within a day. The prime advantages of the biomarker are its efficacy, accuracy and ease of performing analyses both for patient as well as technician. Moreover, cost wise also it is very economical. However, limitation of this biomarker are biased results due to variable urine concentrations, diet factors or urinary tract infection. With the presence of microalbuminuria, National Kidney Foundation (5) classification has suggested that the levels of GFR might be exploited for the accurate staging of chronic kidney disease.

\section{Serum creatinine levels during $\mathrm{DN}$}

One of the most efficient indices used for the determination of clinical health of kidneys is serum creatinine levels (6). The prime route for the metabolism of creatinine produced by 
muscles is via by glomerular filtration. So, during abnormal functioning of kidneys, GFR is affected leading to elevation in the creatinine levels. On the other hand, the only limitation associated with the test that it is unable to detect DN at an early stage. It is applicable only after marked damages to kidney nephrons. Another limitation is that sexes and age of the patient affect serum creatinine levels, so chances of false lows are more in both women and old patients. Cystatin $\mathrm{C}$ is another upcoming indicator of falling GFR (7). A study in the recent past also conformed the dependence of excretion of creatinine on its dietary intake, thus, more the intake the more is the excretion of creatinine (8).

\section{HMGB1}

HMGB1 is a profuse non-histone chromatin, component well known for its dual DNA binding domains viz. HMG box A and B (9). The high affinity of high mobility group box (HMGB)1 for DNA is its characteristic feature. In cancer, elevated levels of HMGB1 are correlated well with cancer progression in the form of invasion and metastasis (10). It is associated with tumor metastasis as it is able to modulate cell adhesive properties and extracellular matrix components (10).

\section{HMGB1, DN and RAGE}

The interaction with a member of immunoglobulin superfamily viz. receptor for advanced glycation end products (RAGE) is the prime pathway for the activation of HMGB1 (11). RAGE is abundant in various tissues and cells including heart, lungs, kidney, brain, skeletal, neutrophils, lymphocytes and monocytes. With advanced glycation end products (AGEs) interaction, RAGE mediates the effects of its other extracellular ligands. Further, RAGE bound HMGB1 indicated the roles of RAGE in cellular migration and intrusiveness (12). Studies in the recent past have confirmed that soluble forms of RAGE might stabilize inflammatory reflexes from RAGE ligands such as AGEs, S100 proteins and HMGB1 $(13,14)$. So, it is another way of HMGB1 to play the role of a cytokine via activation of additional immune responses or inflammatory reactions. Damaged or necrotic cells, leading to inflammation, could also passively release the protein. Further, post-translational modifications such as acetylation, phosphorylation, methylation and poly(ADP)-ribosylation are also responsible for direct HMGB1 action towards secretory pathway (10). All these considerations indicated that HMGB1 is a very important mediator of inflammatory response, with several mechanisms of action not yet completely elucidated. Some recent studies have also shown that release of hyperglycemia-induced HMGB1 leads to renal injury causing tubulointerstitial inflammation during DN (15-17).

\section{HMGB1 signaling and DN}

The prime cellular receptor for HMGB1 is the RAGE that is the main mode of communication between advanced glycation end product (AGE)-modified proteins and the endothelium cells (18). Further, additional pathways viz. CDC42/ Rac pathway and MAPK-NF- $\mathrm{BB}$ pathways are also activated by RAGE binding to HMGB1 (19). Collective activation of the above two pathways often leads to chemotaxis, cytokine activation and migration of immune cells. There are additional receptor(s) relevant to HMGB1 signaling. Consistent with this assumption, follow-up studies further characterized that toll-like receptors 2 (TLR2) and 4, are involved in HMGB1 signaling (12) likely through Rac1/PI3K/CDC42 pathway and MyD88 dependent NF- $\kappa$ B activation pathways.

\section{AGE and DN}

The AGE is the result of a non-enzymatic reaction between reducing monocytes, macrophages and dendritic cells so as to amplify inflammation reaction $(20,21)$. Receptors for AGE (RAGE) when bound to AGEs, associate with reactive oxygen species generation support supplementary AGEs via the nicotinamide adenine dinucleotide phosphate (NADPH) oxidase pathway (22).

The aldose reductase-mediated polyol pathway may directly form AGEs via 3-deoxyglucosone AGE intermediates. This reaction causes depletion of NADPH and glutathione, and the resultant oxidative stress indirectly increases formation of AGEs (21). AGEs can cause extensive cross-linking of proteins and its oxidation, leading to their deposition, as reported in diabetes and Alzheimer's diseases (23). Collectively, these reports indicate that HMGB1's role in the mechanisms of inflammation, apoptosis, angiogenesis, DN and cancer, are very important although still poorly known. Therefore, it is very important to investigate such aspects to better understand these pathogenetic mechanisms and possibly identify the molecular pathways involved, with the aim to develop novel therapeutic strategies for these serious diseases.

\section{Conclusions}

It was concluded from above that diabetic nephropathy is a common outcome associated with diabetes and could prove fatal if not treated in time. Further, the HMGB1 is the key protein responsible for DN and could be exploited as an efficient target for novel therapeutics against DN.

\section{References}

1. Juster-Switlyk K and Smith AG: Updates in diabetic peripheral neuropathy. F1000Res 5: 738, 2016.

2. Wong LP, Li NC, Kansal S, Lacson E Jr, Maddux F, Kessler J, Curd S, Lester K, Herman M and Pulliam J: Urgent peritoneal dialysis starts for ESRD: Initial multicenter experiences in the United States. Am J Kidney Dis 68: 500-502, 2016.

3. Toplak H, Stauber R and Sourij H: EASL-EASD-EASO Clinical Practice Guidelines for the management of non-alcoholic fatty liver disease: Guidelines, clinical reality and health economic aspects. Diabetologia 59: 1148-1149, 2016.

4. Xu HZ, Cheng YL, Wang WN, Wu H, Zhang YY, Zang CS and $\mathrm{Xu} Z \mathrm{ZG}$ : 12-Lipoxygenase inhibition on microalbuminuria in type- 1 and type- 2 diabetes is associated with changes of glomerular angiotensin II type 1 receptor related to insulin resistance. Int J Mol Sci 17: E684, 2016.

5. Jones GRD and Lim EM: The National Kidney Foundation Guideline on estimation of the glomerular filtration rate. Clin Biochem Rev 24: 95-98, 2003.

6. Lakshmanan H, Raman J, Pandian A, Kuppamuthu K, Nanjian R, Sabaratam V and Naidu M: Aqueous extract of Senecio candicans DC induce liver and kidney damage in a sub-chronic oral toxicity study in Wistar rats. Regul Toxicol Pharmacol 79: 25-34, 2016. 
7. Cholongitas E, Ioannidou M, Goulis I, Chalevas P, Ntogramatzi F Athanasiadou Z, Notopoulos A, Alevroudis M, Sinakos E and Akriviadis E: Comparison of creatinine and cystatin formulae with 51 chromium-Edta glomerular filtration rate in patients with decompensated cirrhosis. J Gastroenterol Hepatol 32: 191-198, 2017.

8. Taylor EH: Clinical Chemistry. John Wiley and Sons, New York, NY, pp4 58-62, 1989.

9. Chen Q, Guan X, Zuo X, Wang J and Yin W: The role of high mobility group box 1 (HMGB1) in the pathogenesis of kidney diseases. Acta Pharm Sin B 6: 183-188, 2016.

10. Todorova J and Pasheva E: High mobility group B1 protein interacts with its receptor RAGE in tumor cells but not in normal tissues. Oncol Lett 3: 214-218, 2012.

11. Nagai R, Jinno M, Ichihashi M, Koyama H, Yamamoto $Y$ and Yonei Y: Advanced glycation end products and their receptors as risk factors for aging. Anti-Aging Med 9: 108-113, 2012.

12. Ramasamy R, Yan SF and Schmidt AM: Receptor for AGE (RAGE): signaling mechanisms in the pathogenesis of diabetes and its complications. Ann N Y Acad Sci 1243: 88-102, 2011

13. Sárkány Z, Ikonen T, Ferreira-da-Silva F, Saraiva M, Svergun D, and Damas AM: Solution structure of the soluble receptor for advanced glycation end products (sRAGE). J Biol Chem 286 : 37525-37534, 2011.

14. Per M, Humpert, Djuric Z, Kopf S, Rudofsky G, Morcos M, Nawroth P and Bierhaus A: Soluble RAGE but not endogenous secretory RAGE is associated with albuminuria in patients with type 2 diabetes. Cardiovasc Diabetol 7: 6-9, 2007.

15. Kim J, Sohn E, Kim CS, Jo K and Kim JS: The role of high-mobility group box-1 protein in the development of diabetic nephropathy. Am J Nephrol 33: 524-529, 2011.
16. Lin M, Yiu WH, Wu HJ, Chan LY, Leung JC, Au WS, Chan KW, Lai KN and Tang SC: Toll-like receptor 4 promotes tubular inflammation in diabetic nephropathy. J Am Soc Nephrol 23: 86-102, 2012

17. Penfold SA, Coughlan MT, Patel SK, Srivastava PM, Sourris KC, Steer D, Webster DE, Thomas MC, MacIsaac RJ, Jerums G, et al: Circulating high-molecular-weight RAGE ligands activate pathways implicated in the development of diabetic nephropathy. Kidney Int 78: 287-295, 2010.

18. Hori O, Brett J, Slattery T, Cao R, Zhang J, Chen JX, Nagashima M, Lundh ER, Vijay S, Nitecki D, et al: The receptor for advanced glycation end products (RAGE) is a cellular binding site for amphoterin. Mediation of neurite outgrowth and co-expression of rage and amphoterin in the developing nervous system. J Biol Chem 270: 25752-25761, 1995.

19. Yang H, Wang H, Czura CJ and Tracey KJ: The cytokine activity of HMGB1. J Leukoc Biol 78: 1-8, 2005.

20. Münch G, Keis R, Wessels A, Riederer P, Bahner U, Heidland A, Niwa T, Lemke HD and Schinzel R: Determination of advanced glycation end products in serum by fluorescence spectroscopy and competitive ELISA. Eur J Clin Chem Clin Biochem 35: 669-677, 1997.

21. Huebschmann AG, Regensteiner JG, Vlassara H and Reusch JE: Diabetes and advanced glycoxidation end products. Diabetes Care 29: 1420-1432, 2006.

22. Wautier MP, Chappey O, Corda S, Stern DM, Schmidt AM and Wautier JL: Activation of NADPH oxidase by AGE links oxidant stress to altered gene expression via RAGE. Am J Physiol Endocrinol Metab 280: E685-E694, 2001.

23. Gella A and Durany N: Oxidative stress in Alzheimer disease. Cell Adh Migr 3: 88-93, 2009. 